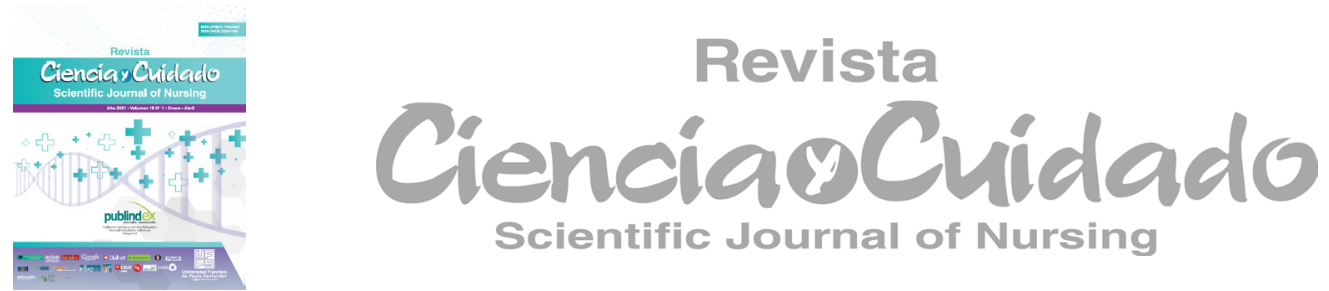

Articulo de Revisión

\title{
Estrategias para fortalecer la humanización de los servicios en salud en urgencias
}

\author{
Strategies to strengthen the humanization of emergency health services.
}

Estratégias para fortalecer a humanização dos serviços de saúde de emergência.

\author{
Erika María Pabón-Ortíz ${ }^{1}$ \\ Jop Vann-Derth Mora-Cruz ${ }^{2}$ \\ Carmen Yaneth Buitrago-Buitrago \\ Ruth Alexandra Castiblanco-Montañez ${ }^{4}$
}

\section{Resumen}

Introducción: La humanización es un factor ético imprescindible y un atributo de la atención en salud. Estudios previos en la institución donde se realizó la investigación se identificaron: la falta de comunicación (80 a 90\%), deficiencia en la capacitación del personal $(52 \%)$, la sobrecarga $(25 \%)$ y el estrés laboral (22\%), como los principales factores relacionados con la deshumanización en la atención de enfermería en urgencias, desde la percepción de los pacientes y del personal. Objetivo: Describir estrategias que fortalezcan el componente de humanización en la prestación de servicios de salud en urgencias de un hospital de cuarto nivel. Metodología: Revisión integrativa en la que se estudiaron 29 investigaciones originales, artículos y literatura gris, publicados en español, inglés y portugués. En su mayoría son indexados y se obtuvieron en revistas de ciencias de la salud mediante las siguientes bases de datos: EBSCOHost, CINAHL, OVID y en el metabuscador de Google Académico; también se tuvieron en cuenta los términos DeCS, MeSH, humanización de la atención, atención de enfermería y urgencias médicas. Es un estudio sin riesgo ético por ser de tipo documental. Resultados: Las estrategias que se reportaron como efectivas y que inciden en los factores relacionados con la falta de humanización son: ausencia de capacitación en competencia de compasión, mindfulness y habilidades blandas; como también, la desinformación en el área de especialización y el aumento de la razón enfermero-paciente. Conclusiones: Las estrategias en mención mejoran la empatía, conciencian el presente, favorecen la comunicación, proporcionan habilidades para el éxito de la atención y disminuyen la sobrecarga laboral.

Palabras clave: Revisión, humanización de la atención, atención de enfermería, urgencias médicas.
Autor de correspondencia*

1* Enfermera. Facultad de Enfermería. Fundación Universitaria de Ciencias de la Salud - FUCS. Bogotá. Correo: empabon@fucsalud.edu.co iD

${ }^{2}$ Enfermero. Facultad de Enfermería. Fundación Universitaria de Ciencias de la Salud - FUCS. Bogotá. Correo: jvmora1@fucsalud.edu.co iD

${ }^{3}$ Enfermera. Especialista en Gerencia en Salud. Instructor Asociado. Facultad de Enfermería. Fundación Universitaria de Ciencias de la Salud - FUCS. Sociedad de Cirugía de Bogotá Hospital de San José - HSJ. Bogotá D.C. Correo: cybuitrago@,fucsalud.edu.co (iD

${ }^{4}$ Enfermera. Magister en Salud Pública. Profesor asistente. Coordinadora de Investigaciones. Facultad de Enfermería. Fundación Universitaria de Ciencias de la Salud - FUCS. Bogotá D.C. Correo: racastiblanco@fucsalud.edu.co (iD)

Recibido: 6 agosto 2020

Aprobado: 15 diciembre 2020

Para citar este articulo:

Pabón-Ortiz EM, Mora-Cruz JV, Buitrago-Buitrago CY, Castiblanco-Montañez RA. Estrategias para fortalecer la humanización de los servicios en salud en urgencias. Rev. cienc. cuidad. 2021;18(1):94-104. https://doi. org/10.22463/17949831.2512

(C) Esta obra está bajo licencia internacional CCBY-NC-ND (c) (1) (9) 


\begin{abstract}
Introduction: Humanization is an indispensable ethical factor and an attribute of health care. Previous studies at the institution where the research was conducted identified: lack of communication (80-90\%), poor staff training (52\%), overload (25\%) and job stress $(22 \%)$ as the main factors related to dehumanisation in emergency nursing care, from the perspective of patients and staff. Objective: To describe strategies that strengthen the humanisation component in the provision of health care in the emergency department of a fourth level hospital. Methodology: Integrative review in which 29 original research studies, articles and grey literature, published in Spanish, English and Portuguese, were studied. Most are indexed and were obtained from health sciences journals using the following databases: EBSCOHost, CINAHL, OVID and in the Google Academic meta-search; the terms DeCS, MeSH, humanisation of care, nursing care and medical emergencies were also taken into account. It is a study without ethical risk as it is of a documentary nature. Results: The strategies reported as effective and that impact on the factors related to the lack of humanisation are: lack of training in compassionate, mindfulness and soft skills competence; as well as, misinformation in the area of specialisation and increased nurse-patient ratio. Conclusions: The strategies mentioned improve empathy, raise awareness of the present, encourage communication, provide skills for successful care and reduce work overload.
\end{abstract}

Keywords: Review, humanization of care, nursing care, medical emergencies.

\title{
Resumo
}

Introdução: A humanização é um fator ético indispensável e um atributo do cuidado com a saúde. Estudos anteriores na instituição onde a pesquisa foi realizada identificaram: falta de comunicação (80-90\%), falta de treinamento do pessoal (52\%), sobrecarga (25\%) e estresse no trabalho $(22 \%)$ como os principais fatores relacionados à desumanização no atendimento de enfermagem de emergência, do ponto de vista dos pacientes e do pessoal. Objetivo: Descrever estratégias que fortaleçam o componente de humanização da prestação de cuidados de saúde de emergência em um hospital de quarto nível. Métodos: Revisão integrativa na qual foram estudadas 29 investigações originais, artigos e literatura cinzenta, publicados em espanhol, inglês e português. A maioria foi indexada e obtida de revistas de ciências da saúde utilizando as seguintes bases de dados: EBSCOHost, CINAHL, OVID e no Google Academic meta-search; os termos DeCS, MeSH, humanização dos cuidados, cuidados de enfermagem e emergências médicas também foram levados em conta. É um estudo sem risco ético porque é de natureza documental. Resultados: As estratégias relatadas como eficazes e que têm impacto sobre os fatores relacionados à falta de humanização são: ausência de treinamento em compaixão, cuidado e competência em habilidades leves; bem como, desinformação na área de especialização e aumento da relação enfermeiro-paciente. Conclusões: As estratégias mencionadas melhoram a empatia, aumentam a consciência do presente, incentivam a comunicação, fornecem habilidades para o cuidado bem sucedido e reduzem a sobrecarga de trabalho.

Palavras-chave: Revisão, humanização dos cuidados, cuidados de enfermagem, emergências médicas.

\section{Introducción}

La humanización es un imperativo ético que debe estar presente en todos los sujetos involucrados en el sistema de salud. Está conformada por todos los actos de cuidado que hacen más humano al hombre, a fin de mantener su dignidad a través de la atención a sus pa- cientes desde una perspectiva holística, integrando las dimensiones psicológica, biológica, social y espiritual del sujeto de cuidado, más allá de considerarse como un componente del proceso de gestión de la calidad de salud o un atributo de la atención. En este sentido hay que tener en cuenta dos culturas: la primera se enfoca en el valor de la eficacia y la segunda, en la prioridad 


\section{Erika María Pabón-Ortíz • Jop Vann-Derth Mora-Cruz • Carmen Yaneth-Buitrago • Ruth Alexandra Castiblanco-}

Montañez

al respeto por la persona, su proceder y la preservación de sus derechos. Por otro lado, la humanización es un eje de acreditación que busca suplir las necesidades de los usuarios (1-5).

Contrario a la humanización, está la deshumanización, entendida como el desenfoque y cosificación del ser, lo que acarrea consecuencias tales como: baja realización laboral, comunicación deficiente y recorte de personal para los enfermeros; por otro lado, es frecuente la omisión de diagnósticos y terapéuticas, la percepción de la atención es de baja calidad, se alarga el tiempo de recuperación y la estancia de los pacientes (6). Del mismo modo, existen diferentes factores que influyen en la deshumanización como lo son: tiempos de espera prolongados, algunos componentes organizacionales, se afecta la salud mental del personal, hay ineficacia en la comunicación enfermero-paciente, insensibilidad durante los procedimientos invasivos, asesoramiento superficial, falta de empatía y de rapidez, etc.. Factores que se agudizan en el área de urgencias, por la insuficiencia de recursos humanos frente a la demanda de pacientes, especialmente en una Institución Prestadora de Servicios de Salud (IPS); igualmente afectan la satisfacción del paciente, conformada por las expectativas de atención idónea frente a su percepción posterior (7-14).

Conviene indicar que en una institución de cuarto nivel se han identificado, por una parte, la perspectiva de los pacientes frente a los comportamientos del cuidado humanizado de enfermería; y, por la otra. la percepción del personal asistencial y los factores que influyen en la atención humanizada.

En lo que concierne a la perspectiva de los pacientes acerca de los factores que no permiten un cuidado humanizado de enfermería, se reportó que los que más influyen son: no se dedica tiempo para aclarar las inquietudes del usuario, en un $82 \%$; el personal de salud no se preocupa por el estado de ánimo del paciente, en un $83 \%$, es decir, que no se está atendiendo al sujeto de cuidado como un ser biopsicosocial. Respecto a los factores que dificultan la atención humanizada según el personal asistencial, lo que más los afecta son: la sobrecarga laboral que impide dedicarle tiempo a los pacientes, debido al estrés que genera la posibilidad de no terminar todas las actividades de las que son responsables al finalizar el turno, manifestado por un $22 \%$; la incongruencia entre los deberes a realizar y el tiempo para ejecutarlos, según el $25 \%$; y, por último, una eficiente capacitación para brindar un cuidado humaniza- do, al dedicarse a cumplir exclusivamente las tareas de las que son responsables, sustentado por un $52 \%$ de las respuestas $(15,16)$.

Aunque la institución mencionada cuenta con políticas y programas de humanización, no se dispone de medidas que subsanen los factores mencionados; por tanto, es necesario crear estrategias que propicien ambientes más tranquilos para los pacientes, disminuyendo sus niveles de estrés, lo que permitirá que el personal esté más sereno. Dichas estrategias deberán estar coordinadas por un comité del hospital, que se encargue de establecer acciones para optimizar el ambiente laboral, generando así una atención humanizada al paciente y a la familia $(8,15-17)$.

Se debe subrayar, que frente a una situación clínica de urgencia hay que instaurar una intervención inmediata que responda a la necesidad identificada, teniendo en cuenta la posición de vulnerabilidad y la dependencia que tienen los pacientes del personal de salud, sobretodo del enfermero/a, quien ejerce un cuidado directo. Según lo reportado por la literatura, la satisfacción de los sujetos de cuidado en términos de humanización ha cubierto en su mayoría las expectativas; no osbtante, la buena impresión se ha asociado a que la condición de salud es tan frágil que cualquier asistencia del personal es apreciada positivamente y en realidad, la percepción de la atención humanizada no es del 100\%. Al respecto, las instituciones intentan poner en práctica políticas y programas que humanicen la atención en el área de urgencias, implementando propuestas como: involucrar al paciente durante el triage, para que comprenda la decisión sobre la prioridad de intervención de su condición; incrementar los recursos humanos, para que ayuden en las tareas que impiden a los enfermeros realizar un cuidado dedicado y sensible, como el traslado de pacientes o el control de los tiempos de espera; y, aplicar musicoterapia, etc. En este sentido las dos primeras actividades, generan resultados positivos y la última, actúa como relajante; sin embargo, la deshumanización continúa siendo una problemática de diversas etiologías. Por tanto, la propuesta es continuar investigando los factores desencadenantes (17-20).

Ante la evidente presencia de atención deshumanizada, es de vital importancia determinar cuáles son los aspectos más afectados y a partir de ahí, desarrollar estrategias para intervenirlos. Así, tanto el personal de enfermería como el sujeto de cuidado se benefician de la humanización, en la medida en que se disminuya el estrés de los profesionales, permitiendo que la curación 
de los pacientes se acelere; además, la comunicación es más efectiva y por tanto será percibida de forma positiva por los usuarios, lo que hará que su actitud sea apacible, preservándose la tranquilidad del personal, entre otras $(7,15,16,18,19)$.

Por todo lo planteado anteriormente, esta investigación busca describir las estrategias que fortalezcan el componente de humanización en la prestación del servicio de salud en urgencias de un hospital de cuarto nivel.

\section{Metodología}

Se realizó una revisión integrativa de la literatura siguiendo los seis pasos propuestos por Méndez, Silveira y Galvão (21), los cuales posibilitaron el planteamiento del problema y el desarrollo de la pregunta de investigación de acuerdo al acrónimo PICO: ¿Cuáles son las acciones que fortalecen el componente de humanización en la prestación del servicio de salud? Luego se elaboraron las ecuaciones de búsqueda en los idiomas español, inglés y portugués a partir de los términos DeCS y MeSH: Humanización de la atención, atención de enfermería y urgencias médicas, junto con los operadores booleanos AND y OR. Dentro de los criterios de inclusión se tuvieron en cuenta publicaciones originales de investigación primaria y secundaria, divulgadas en el periodo de 2007 a 2020. La búsqueda se realizó en las siguientes bases de datos: EBSCOHost, CINAHL, OVID, Scielo, ClinicalKey, CIBERINDEX, Redalyc, Business Source Complete y Biblioteca Virtual en Salud, incluidas en el catálogo de la Fundación Universitaria de Ciencias de la Salud (FUCS) y en Google Académico. El proceso de búsqueda se efectuó entre septiembre de 2019 y abril de 2020; al respecto se analizaron 29 investigaciones materializadas en artículos y literatura gris.

Por otra parte, se filtraron los artículos por título, resumen y texto completo, que respondían al objetivo de la revisión. A continuación, la información extraída de las unidades de análisis definitivas se organizó por temáticas, conformadas por la reducción y síntesis de los datos relevantes, en una matriz construida en Microsoft Excel®. Estos datos se relacionaban con las estrategias que impactan en los factores que desencadenan la falta de humanización.

Del mismo modo, se profundizó sobre la exploración de las estrategias para conformar el estado del conocimiento y así determinar el nivel de evidencia, para ofrecer recomendaciones. Desde este punto de vista, se incluyeron todos los reportes de investigaciones disponibles al respecto. Igualmente, se utilizaron Parrillas CASPe para ensayos clínicos y revisiones sistemáticas, Agree II para guías de práctica clínica y Strobe para los diseños de corte transversal y observacionales.

Esta investigación comprende y obedece a las consideraciones éticas dadas por el Ministerio de salud de Colombia en la Resolución 8430 de 1993, que establece las normas científicas, técnicas y administrativas para la investigación en salud, clasificándola como una investigación sin riesgo por ser de tipo documental (22) y en la Ley 44 de 1993 que reglamenta la protección de los derechos del autor (23).

\section{Análisis}

\section{Aspectos formales de las unidades de análisis:}

En la búsqueda inicial se hallaron 169 artículos que se limitaron a 29 definitivos que responden al objetivo del estudio. Se resalta que el $24,13 \%$ de las investigaciones fueron publicadas en Colombia, por profesionales de la salud (ver figura 1).

Conviene subrayar que los países con mayor publicación fueron: Colombia, España y Chile, seguidos de Perú, México, Ecuador, Brasil, Singapur y Estados Unidos (ver tabla 1$)$.

Tabla 1. Paises con mayor publicacion

\begin{tabular}{|cc|}
\hline País & Cantidad de unidades de análisis finales \\
\hline Colombia & 7 \\
\hline España & 6 \\
\hline Chile & 5 \\
\hline Perú & 3 \\
\hline México & 3 \\
\hline Ecuador & 2 \\
\hline Brasil & 1 \\
\hline Singapur & 1 \\
\hline Estados Unidos & 1 \\
\hline
\end{tabular}

Fuente: Autores 
Erika María Pabón-Ortíz • Jop Vann-Derth Mora-Cruz • Carmen Yaneth-Buitrago • Ruth Alexandra CastiblancoMontañez

Los resultados se sintetizaron en cinco estrategias efectivas para reforzar el componente de humanización de la atención en salud: competencia de compasión, Mindfulness, fortalecimiento de habilidades blandas, formación del personal en su área de especialización y mejora de la razón enfermero-paciente. A continuación, se describen al inicio de cada una de las estrategias, los factores que más inciden identificados en estudios anteriores sobre análisis de humanización, desde la percepción de los pacientes y del personal de enfermería en el servicio de urgencias de una institución de cuarto nivel. Posteriormente, se presenta su fundamentación $(15,16)$.

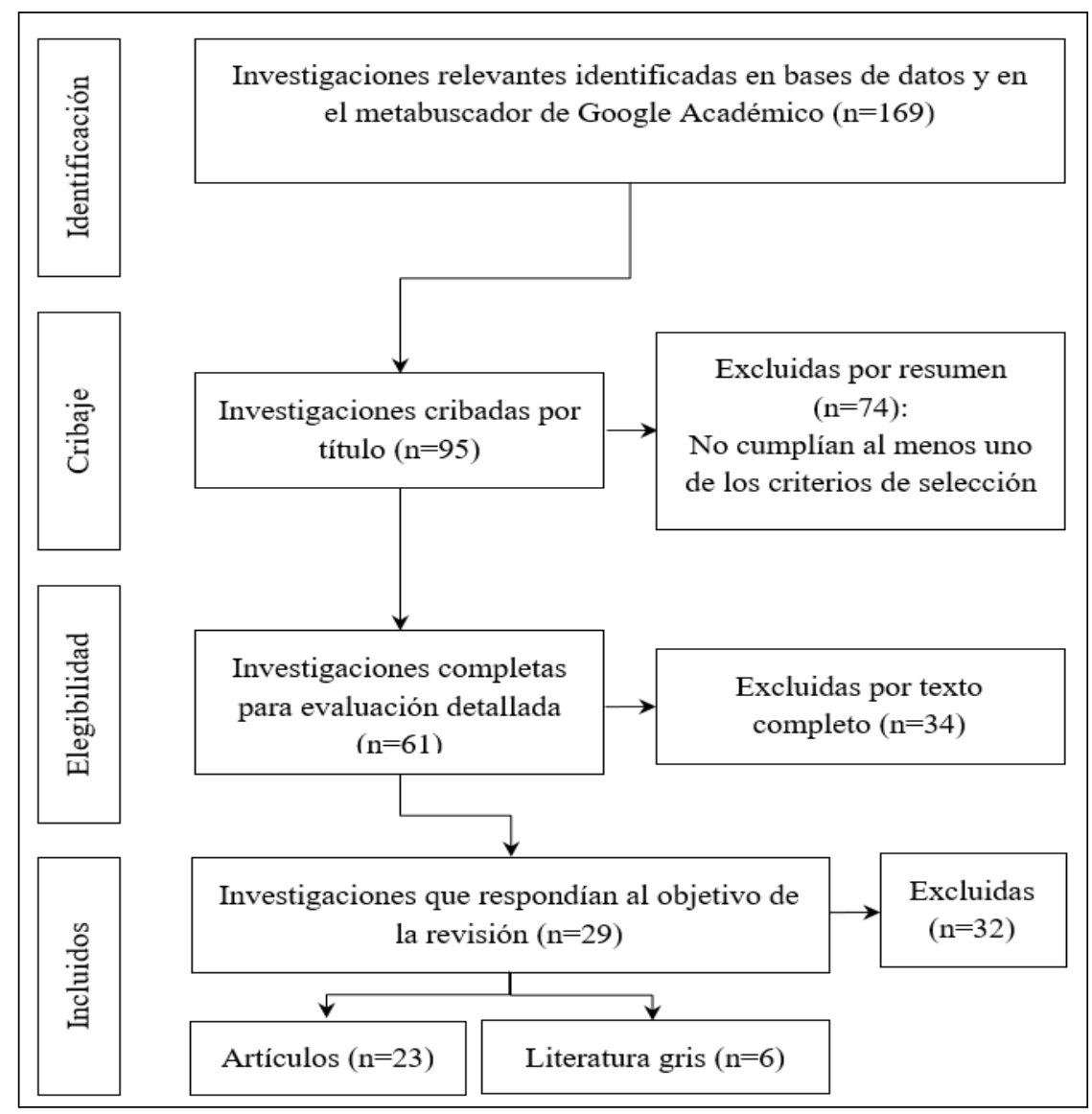

Figura 1. Proceso de selección de unidades de análisis finales. Fuente: Autores

\section{Estrategia 1: Competencia de compasión.}

Por medio del instrumento sobre percepción de comportamientos de cuidado humanizado de enfermería (PCHE), se identificó que los factores afectados, relacionados con la priorización del sujeto de cuidado, impactan por medio de la aplicación de la competencia de compasión. Esta estrategia fue fundamentada por 6 unidades de análisis $(15,24-28)$.

La compasión es una de las competencias que más interviene en el cuidado directo de los pacientes y ayuda a la creación de vínculos entre el personal de enfermería y sus sujetos de cuidado, lo que permite satisfacer diferentes necesidades del tipo emocional y acelera la recuperación de los pacientes (26). Según un estudio cualitativo que utilizó un diseño etnográfico sobre La compasión como valor ético del profesional enfermero, la aplicación de la compasión es esencial en el alcance de la atención de alta calidad y de cuidados virtuosos, al generar en el colaborador conmoción por el sufrimiento de los pacientes (27).

Por otro lado, la competencia sobre la compasión permite identificar y comprender el sufrimiento del otro y ayuda a mejorar el deseo de mitigar y acabar con el dolor, ya que se promueven acciones con amor, comprensión, empatía, ayuda y motivación; actitudes que 
son importantes en cada momento de la vida de los sujetos de cuidado (28).

Por consiguiente, la compasión debe ser una competencia fundamental en el ejercicio del profesional de enfermería, puesto que repercute en la calidad de la atención que percibe el sujeto de cuidado y a su vez favorece su evolución, que con frecuencia es positiva.

\section{Estrategia 2: Mindfulness.}

Partiendo de la premisa por la cual se plantea que el personal de enfermería presenta estrés laboral, se recomienda el uso del Mindfulness, estrategia soportada en 6 artículos $(16,29-33)$.

Mindfulness está basada en la atención plena a los estímulos internos y externos del presente, contribuyendo al constructo de experiencias vividas sobre curiosidad y aceptación (29). En este sentido, a partir de la evaluación de los propios procesos cognitivos (30), que al aplicarlos no tendrán juicios de valor, se estará atento a la percepción y el sentimiento; es decir, que se evita el centrar la atención en preocuparse por lo que sucedió o sucederá, ya que se está enfocado en el aquí y el ahora.

Desde otro punto de vista, el Mindfulness, es descrito por la literatura como un estado metacognitivo, con el que se logra moderar los pensamientos de angustia que distraen; es decir, que las acciones pasadas no influyen y se pueden revaluar de manera positiva. Este enfoque da como resultado emociones positivas relacionadas con el amor, la compasión y la confianza (31).

Siendo así, se recomienda la implementación de un programa que eduque a los colaboradores en el manejo de sus emociones. Al respecto un ensayo clínico controlado y aleatorio que aplicó una intervención basada en Mindfulness y autocompasión a profesionales de la enfermería y la medicina, quienes no habían empleado dichas prácticas en los últimos 6 meses, dio como resultado una puntuación media preintervención en Mindfulness de 120,5 (DE: 19,5), que mejoró significativamente a 140,1 (DE: 14,2) tras la intervención (32). En otro estudio de Evaluación de la efectividad de un programa de Mindfulness en profesionales de atención primaria, un $89 \%$ de los participantes ya ejecutaban la técnica por sí solos y un $94 \%$ indicó que sus habilidades de autocuidado habían progresado y que eran mejores profesionales. En definitiva, el uso y aplicación de un programa basado en Mindfulness mejora la habilidad de la empatía (33).

De donde se infiere que en su ejercicio el profesional de enfermería, quien es responsable de diversas tareas, no solamente del área asistencial sino de la administrativa, es determinante enfocar su atención en el presente, para que le sea posible ejercer su máximo potencial y a su vez, brindar una atención óptima.

\section{Estrategia 3: Fortalecimiento de habilidades blan-} das.

Dada la falta de comunicación encontrada con la aplicación del instrumento PCHE, se propone el fortalecimiento de las habilidades blandas; al respecto, 7 investigaciones aportaron elementos para la construcción de esta estrategia $(15,34-39)$.

Las habilidades blandas son atributos de una persona que le permiten interactuar de manera asertiva y con un nivel alto de comunicación; además, le facilitan la adaptación, sus comportamientos son efectivos y mejoran la calidad de la atención. Se ha demostrado que este tipo de habilidades son necesarias en la formación de las personas, ya que añaden valor en el campo de la acción laboral, mediante un desempeño exitoso en el trabajo, al mejorar el trato humano de quienes las aplican; acciones que deben estar directamente relacionadas con los resultados esperados por una entidad $(34,35)$. Dichas destrezas contribuyen en todo el proceso de intervención en la salud, puesto que mejoran el cumplimento del plan terapéutico, así como el alcance del tratamiento. Al respecto, las habilidades presentes en los enfermeros son: la cordialidad, la amabilidad, la calidez, la paciencia, la tolerancia, etc. (36-38).

Sobre el particular, se fomentará la aplicación de las habilidades blandas que generen sensibilización y empatía en el personal, pues en los servicios se requiere del uso de estas destrezas para que los pacientes se sientan en un entorno confortable y seguro (39).

En definitiva, el fortalecimiento de las habilidades blandas en los enfermeros, repercutirá en la adaptación y sensación de plenitud del sujeto de cuidado, ya que favorece la interacción asertiva entre ambos.

Por consiguiente, es de gran importancia gestionar la contratación de expertos en la competencia de compasión, Mindfulness y habilidades blandas, quienes facilitarán la calidad de la atención evitando el estrés laboral con una mejor comunicación. Este tipo de intervención se focaliza especialmente en el mejoramiento de la humanización en urgencias.

Estrategia 4: Formación del personal en su área de especialización. 


\section{Erika María Pabón-Ortíz • Jop Vann-Derth Mora-Cruz • Carmen Yaneth-Buitrago • Ruth Alexandra Castiblanco-}

Montañez

De las 29 unidades de análisis finales, 5 evaluaron esta estrategia a través de su investigación $(27,40-43)$. Con respecto a este tópico, un estudio realizado en la Universidad de Chile menciona los aspectos generales de la gestión de los servicios de urgencias para la mejora de la atención de los pacientes, señalando que se debe contar con recurso humano capacitado en el área, ya que conduce al éxito de la unidad; además, debe ejecutarse a partir de un proceso de planificación que seleccione el personal con capacidades técnicas y concomitantemente con habilidades relacionadas (40). Adicionalmente, un estudio descriptivo que buscaba determinar los factores relacionados con la calidad de la atención en enfermería, encontró que: el $92.96 \%$ del personal de enfermería entrevistado estaba capacitado; la relación enfermero-paciente es del 76.06\%; y, el conocimiento y aplicabilidad de procedimientos en el $25.35 \%$.

Por otro lado, dicho estudio determinó que la capacitación debe ser continua conforme al servicio asignado, a los procedimientos asistenciales del área y a los aparatos electromédicos; sobre todo hay que mejorar la calidad de la atención. De igual forma, se debe continuar con la interiorización del proceso de atención en la enfermería en función de las siguientes taxonomías: NANDA International, Inc (NANDA-I), Nursing Outcomes Classification (NOC) y Nursing Interventions Classification (NIC) (41).

Al mismo tiempo, otro estudio que buscaba describir los factores que influyen en la sobrecarga de los cuidadores de adultos mayores concluyó, utilizando la escala Zarit, que la mayoría de los cuidadores encuestados, correspondiente al $64,5 \%$, no presentaban sobrecarga puesto que eran colaboradores formales; además, recibían capacitación, que les brinda herramientas y los concientiza sobre las problemáticas de la labor, tales como la sobrecarga; más cuando se sabe que la falta de preparación es la causante de distintos niveles de sobrecarga laboral (42).

Es necesario recalcar que la excelencia de los profesionales de la enfermería está enfocada en obtener la experiencia y el conocimiento necesarios en el área del saber en el que se encuentran. Al respecto, Patricia Benner, enfermera teorista quien indagó sobre el concepto de desarrollo profesional de novato a experto, a través de la adquisición de competencias, planteó que en el proceso transcurrido desde la graduación de enfermería hasta la especialización del profesional, surgen cambios conductuales, que están relacionados con la integración del conocimiento y la experiencia.
Igualmente, se consiguen habilidades que permiten que el desempeño profesional sea mejor, impactando en la calidad de la atención y el cuidado que, según la autora, es proporcional al nivel de formación (27). Por lo anterior, se recomienda formar al personal de enfermería en su área de especialización para así mejorar la calidad de la atención (43).

Indiscutiblemente la formación en el área de la especialización potenciara en el profesional de la enfermería las habilidades del "saber-hacer" y en consecuencia, las del "ser", impactando directamente sobre la humanización del cuidado directo al paciente, respondiendo a sus necesidades y prioridades.

\section{Estrategia 5: Mejora de la razón enfermero-pacien- te}

Esta estrategia está justificada por 7 estudios (44-50), dentro de los cuales se halló en una tesis de sobrecarga laboral en profesionales de Enfermería, realizada en la Universidad Nacional de Colombia, que las labores asistenciales sumadas a las administrativas limitan el tiempo para su realización, debido a que la sobrecarga es un factor que afecta la eficiencia y la clase de la atención, la forma de asistencia y la calidad del acceso (44). Sin embargo, como lo indica la literatura existente la cantidad media de pacientes atendidos, junto a un número adecuado de recursos humanos logra optimizar las responsabilidades del trabajo, impactando positivamente sobre la calidad de la atención y la seguridad tanto de los pacientes como del personal (45). Adicionalmente, es la dimensión administrativa la que incide sobre el trabajo, puesto que exige al profesional encargarse de la organización y la gestión de los recursos humanos.

Considerando que la razón enfermero-paciente de unidades de atención crítica como urgencias y cuidados intensivos es de 1:2 o 3, debido a la complejidad de dichos servicios, se recomienda aumentar la contratación de personal especializado para reducir la carga de quienes trabajan allí. Por el contrario, en un estudio longitudinal multicéntrico de España se demostró que entre más elevada sea la razón enfermero-paciente, mayor es el riesgo de mortalidad; es por eso, que cuando la cantidad de pacientes era mayor a 2,5 la mortalidad aumentaba en un 3,5\% (46). En cambio, en una revisión sistemática se demostró que el incremento de una enfermera a tiempo completo en la Unidad de Cuidados Intensivos (UCI) disminuyó la probabilidad de morir en un 9\%; los efectos adversos tuvieron un de- 
crecimiento del $60 \%$ en falla respiratoria; $51 \%$ en extubación accidental; un 30\% en aparición de neumonías y un $28 \%$ en parada cardíaca $(47-49,50)$.

Por lo tanto, hay que mejorar la ratio enfermero-paciente, ya que asegurará una mayor disponibilidad de la enfermería en la atención de sus sujetos de cuidado y disminuirá los fallos que comprometan la salud o propicien indirectamente su deterioro.

\section{Conclusiones}

- Las competencias relacionadas con la compasión, el Mindfulness y las habilidades blandas han demostrado generar, según la literatura, cuidados asertivos que favorecen el proceso de recuperación al suplir las necesidades emocionales de los sujetos de cuidado. También sirven para la orientación hacia las situaciones actuales moderando las distracciones, los comportamientos y las relaciones efectivas basadas en la empatía. Por consiguiente las IPS deben aplicar estrategias que beneficien la calidad de la atención en salud reforzando el componente de humanización, considerándolo como uno de los principales elementos dentro del plan de cuidados, necesario para lograr una intervención holística.
- Por otro lado, la formación del personal en el área de especialización y el mejoramiento de la razón enfermero-paciente son determinantes en el perfeccionamiento de la atención, al disminuir la exposición a la sobrecarga laboral. Todo lo planteado anteriormente se logrará a través del fortalecimiento de los saberes y la optimización del talento humano; además, se deberá propiciar la preservación del bienestar de los pacientes y del personal de salud, disminuyendo la presencia de eventos adversos. Por tanto, es indispensable que el sector administrativo tramite lo mencionado para el beneficio de la calidad del servicio de salud en términos de humanización.

- Las estrategias mencionadas han demostrado a través de la evidencia, que son efectivas para mejorar el componente de humanización; por consiguiente, se recomienda emplearlas en los servicios de salud y evaluar su impacto.

\section{Conflicto de Intereses}

Los autores declaran no tenen ningún conflicto de interés.

\section{Referencias Bibliográficas}

1. Correa-Zambrano ML. La humanización de la atención en los servicios de salud: Un asunto de cuidado. Rev Cuid. 2016; 7(1): 1227-1231. https://doi.org/10.15649/cuidarte.v7i1.300

2. Díaz-Rodríguez M, Alcántara-Rubio L, Aguilar-García D, Puertas-Cristóbal E, Cano-Valera M. Training guidelines for humanised nursing care: an integrative literature review. Enferm Global -Esp-. 2020; 58: 657-672. https://doi.org/10.6018/eglobal.392321

3. Bautista-Rodríguez LM, Arias-Velandia MF, Carreño-Leiva ZO. Percepción de los familiares de pacientes críticos hospitalizados respecto a la comunicación y apoyo emocional. Rev Cuid. 2016; 7(2): 1297-1309. https://doi.org/10.15649/cuidarte.v7i2.330

4. Cano SM, Giraldo A, Forero C. Concepto de calidad en salud: Resultado de las experiencias de la atención, Medellín, Colombia. Rev Fac Nac Salúd Pública. 2016; 34(1): 48-53. https://doi.org/10.17533/udea.rfnsp. v34n1a06

5. Instituto Colombiano de Normas Técnicas y Certificación [Sede Web]. Colombia: Dirección de Acreditación en Salud; 2016 [Consultado 16 de septiembre de 2019]. Humanización de la atención; [Aproximadamente 2 pantallas]. Disponible en: http://www.acreditacionensalud.org.co/ea/Paginas/HumAte.aspx

6. Bueno MC, Girón MC, Mesa CM, Moscote DE. ¿Qué favorece la no humanización en nuestro sistema de salud? [Tesis de grado] Cali: Universidad Católica de Manizales; 2014. [Consultado 16 de septiembre de 2019] Disponible en: http://repositorio.ucm.edu.co:8080/jspui/bitstream/handle/10839/1059/Martha\%20Cecilia $\% 20$ Bueno $\% 20$ Garcia.pdf? sequence $=1 \&$ isAllowed $=y$

7. Barbosa-Rodríguez JM. Diseño de un sistema de indicadores para evaluar la eficiencia de los procesos en el servicio de urgencias. [Tesis de grado] Bogotá D.C.: Universidad Distrital Francisco José de Caldas; 2017. [Consultado 16 de septiembre de 2019] Disponible en: http://repository.udistrital.edu.co/bitstream/11349/6384/2/ 
Erika María Pabón-Ortíz • Jop Vann-Derth Mora-Cruz • Carmen Yaneth-Buitrago • Ruth Alexandra CastiblancoMontañez

BarbosaRodr\%C3\%ADguezJuanManuel2017.pdf

8. Arteta-Poveda LE, Palacio-Salgar K. Dimensiones para la medición de la satisfacción del paciente en centros de urgencia. Rev. salud pública. 2018; 20(5): 629-636. https://doi.org/10.15446/rsap.v20n5.61032

9. Joven ZM, Guáqueta-Parada SR. Percepción del paciente crítico sobre los comportamientos de cuidado humanizado de enfermería. Av Enferm. 2019; 37(1): 65-74. https://doi.org/10.15446/av.enferm.v37n1.65646

10. Valenzuela-Anguita M, Sanjuan-Quiles A, Ríos-Risquez MI, Valenzuela-Anguita MC, Juliá-Sanchis R, Montejano-Lozoya R. Humanization of healthcare at the emergency department: A qualitative analysis based on nurses' experiences. Referência. 2019: 4(23): 59-68. https://doi.org/10.12707/RIV19030

11. Pares-de-Oliveira P, Bezerra Rodrigues-A, Gimenez-Amaral J, Dos-Santos-Kubo M, Araújo-da-Silveira EA. A humanização da assistência na ótica de profissionais de enfermagem que cuidam de idosos. Inv Enf. 2018; 20(2): 1-12. https://doi.org/10.11144/Javeriana.ie20-2.haop

12. Romero-Massa E, Contreras-Méndez I, Moncada-Serrano A. Relación entre cuidado humanizado por enfermería con la hospitalización de pacientes. Hacia Promoc Salud. 2016; 21(1): 26-36. https://doi.org/10.17151/ hpsal.2016.21.1.3

13. Beltrán-Salazar OA. The meaning of humanized nursing care for those participating in it: Importance of efforts of nurses and healthcare institutions. Invest Educ Enferm. 2016; 34(1): 18-28. https://doi.org/10.17533/udea. iee.v34n1a03

14. Beltrán-Salazar OA. Impersonal Care or Humanized Care: A Decision Made by Nurses? Hourglass Model. Invest Educ Enferm. 2016; 34(3): 444-455. https://doi.org/10.17533/udea.iee.v34n3a03

15. Buitrago CY, Castiblanco-Montañez RA, Cardona-Endo YK, Galindo-Pérez AL, Guzmán-Bustacara JP, Rodríguez-Bolívar SR. Percepción de los pacientes sobre los comportamientos del cuidado humanizado de enfermería en el servicio de urgencias adultos del Hospital San José. 2018.

16. Buitrago CY, Castiblanco-Montañez RA, Sarmiento-Jaimes LA, Pinillos-Rodriguez E. Percepción del personal asistencial sobre los factores que influyen en la atención humanizada. 2019.

17. Batista-Paula CF, Helu-M-Ribeiro RC, Lins-Werneck A. Humanization of care: Reception and screening in risk classification. J Nurs UFPE online. 2019; 13(4): 997-1005. https://doi.org/10.5205/1981-8963v13i04a238728p997-1005-2019

18. Sánchez-Bolívar MA. Identificación de estrategias para la humanización y calidad en la prestación de los servicios de salud en Bogotá Colombia. [Tesis de grado] Bogotá D.C: Universidad Militar Nueva Granada; 2017. [Consultado 16 de septiembre de 2019] Disponible en: https://repository.unimilitar.edu.co/bitstream/ handle/10654/15811/SanchezBolivarMayraAlejandra2016.pdf; sequence=3

19. García-García I. La humanización de la asistencia en los servicios de urgencias. [Tesis de grado] Madrid: Universidad Autónoma de Madrid; 2018. [Consultado 20 de noviembre de 2019] Disponible en: https://repositorio.uam.es/bitstream/handle/10486/684868/garcia garcia irenetfg.pdf? sequence=1\&isAllowed=y

20. Feitosa-Sousa KHJ, Sena-Damasceno CKC, Landim-Almeida CAP, Macêdo-Magalhães J, De Assunção Ferreira M. Humanization in urgent and emergency services: contributions to nursing care. Rev Gaúcha Enferm. 2019; 40:e20180263. https://doi.org/10.1590/1983-1447.2019.20180263

21. Dal-Sasso-Mendes K, De-Campos-Pereira-Silveira RC, Galvão CM. Revisão integrativa: método de pesquisa para a incorporação de evidências na saúde e na enfermagem. Texto Contexto Enferm. 2008; 17(4): 758-764. https://doi.org/10.1590/S0104-07072008000400018

22. Resolución número 8430 de 1993. Por la cual se establecen las normas científicas, técnicas y administrativas para la investigación en salud. (4 de octubre de 1993). [Consultado 20 de noviembre de 2019] Disponible en: https://www.minsalud.gov.co/sites/rid/Lists/BibliotecaDigital/RIDE/DE/DIJ/RESOLUCION-8430-DE-1993. $\underline{\mathrm{PDF}}$

23. Ley 44 de 1993. Por la cual se modifica y adiciona la ley 23 de 1982 y se modifica la ley 29 de de 1944 . (5 de febrero de 1993). [Consultado 20 de noviembre de 2019] Disponible en: http://derechodeautor.gov.co/documents/10181/182597/44.pdf/7875d74e-b3ef-4a8a-8661-704823b871b5

24. González-Hernández OJ. Validez y confiabilidad del instrumento 'Percepción de comportamientos de cuidado humanizado de enfermería PCHE $3^{\text {a }}$ versión”. Aquichán. 2015; 15(3): 381-392. https://doi.org/10.5294/ aqui.2015.15.3.6 
25. Soares-Rezende-Lopes MT, Gomes Labegalini CM, Antoniassi Baldissera VD. Educar para humanizar: o papel transformador da educação permanente na humanização da atenção básica. Rev enferm UERJ. 2017; 25:e26278. https://doi.org/10.12957/reuerj.2017.26278

26. Retamoza PL. Dimensión espiritual del cuidado de enfermería para el cliente hospitalizado. [Tesis doctoral] Trujillo: Universidad Nacional de Trujillo; 2016 [Consultado 20 de noviembre de 2019] Disponible en: http:// dspace.unitru.edu.pe/bitstream/handle/UNITRU/5898/TESIS\%20DOCTORAL\%20PATRICIA\%20DE\%20 LOURDES\%20RETAMOZA.pdf? sequence=1\&isAllowed=y

27. Bueno Arnau MI. Compasión, valor ético del profesional enfermero: Estudio etnográfico en el área de salud de Soria. [Tesis de grado] Barcelona: Universidad de Barcelona; 2018. [Consultado 20 de noviembre de 2019] Disponible en: http://diposit.ub.edu/dspace/bitstream/2445/124008/1/MIBA_TESIS.pdf

28. Librada-Flores S. Grupo de trabajo de la SECPAL de comunidades compasivas al final de la vida. Med Paliat. 2017; 24(3): 115-116. https://doi.org/10.1016/j.medipa.2017.05.001

29. Moñivas A, García-Diex G, García-de-Silva R. Mindfulness (atención plena): Concepto y teoría. Portularia. 2012; 7(extra): 83-89. https://doi.org/10.5218/prts.2012.0009

30. Osses S, Jaramillo S. Metacognición: Un camino para aprender a aprender. Estud. pedagóg. 2008; 34(1): $187-$ 197. https://doi.org/10.4067/S0718-07052008000100011

31. Moscoso MS. Mindfulness, autoregulación emocional y reducción del estrés: ¿ciencia o ficción? RMIP. [Revista en internet] 2018 [Consultado 20 de noviembre de 2019]; 10(2): 101-121.. Disponible en: https://www. medigraphic.com/pdfs/revmexinvpsi/mip-2018/mip182a.pdf

32. Aranda-Auserón G, Elcuaz Viscarret MR, Fuertes-Goñi C, Güeto-Rubio V, Pascual-Pascual P, Sainz-de-Murieta E. Evaluación de la efectividad de un programa de mindfulness y autocompasión para reducir el estrés y prevenir el burnout en profesionales sanitarios de atención primaria. Aten Prim. 2018; 50(3): 141-150. https:// doi.org/10.1016/j.aprim.2017.03.009

33. Martín-Asuero A, Rodríguez-Blanco T, Pujol-Ribera E, Berenguera A, Moix-Queraltó J. Evaluación de la efectividad de un programa de mindfulness en profesionales de atención primaria. Gac. sanit. 2013; 27(6): 521-528. https://doi.org/10.1016/j.gaceta.2013.04.007

34. Vera-Millalén F. Infusión de habilidades blandas en el currículo de la educación superior: clave para el desarrollo de capital humano avanzado. Akademéia [Revista en internet]. 2016 [Consultado 20 de noviembre de 2019]; 7(1): 53-73. Disponible en: http://repositorio.ugm.cl/bitstream/handle/12345/1407/Infusion\%20 de $\% 20$ habilidades.pdf? sequence $=1 \&$ isAllowed $=y$

35. Ortega-Santos CE, Febles-Rodríguez JP, Estrada-Sentí V. Una estrategia para la formación de competencias blandas desde edades tempranas. Rev. cuba. educ. super [Revista en internet]. 2016 [Consultado 20 de noviembre de 2019]; 2: 35-41. Disponible en: https://pdfs.semanticscholar.org/d7b8/019e223ad368c169f0733cc89bae1706fd60.pdf? ga=2.138553782.46494750.1591558092-187085161.1591558092

36. Lagos-Sánchez Z, De-Mattos-Pimenta CA, Urrutia-Soto MT. Competencias blandas y calidez en enfermería: definiciones, concepción y características. Horiz. enferm [Revista en internet]. 2013 [Consultado 20 de noviembre de 2019]; 24(1): 32-41. Disponible en: http://horizonteenfermeria.uc.cl/images/pdf/24-1/31_competencias $\% 20$ blandas $\% 20 \mathrm{y} \% 20$ calidez $\% 20$ en $\% 20$ enfermera $\% 20$ definiciones $\% 20$ concepcin $\% 20 \mathrm{y} \% 20$ caracteristicas_horizonte $\% 2024-12013 . p d f$

37. Majid S, Eapen CM, Oo KT, Aung EM. The Importance of Soft Skills for Employability and Career Development: Students and Employers' Perspectives. IUP. 2019; 13 (4): 7-39. Disponible en: https://www.questia. com/library/journal/1P4-2330760547/the-importance-of-soft-skills-for-employability-and

38. Lazo-Moreira MV, Véliz-Santos KA. Las habilidades blandas del psicólogo clínico en su intervención en la salud pública. Contrib. cienc. soc [Revista en internet]. 2017 [Consultado 10 de enero de 2020]. Disponible en: http://www.eumed.net/rev/cccss/2017/03/psicologo-clinico-ecuador.html

39. Astochado-Granados TT. Habilidades blandas y calidad de atención en el Hospital de Apoyo II Gustavo Lanatta Luján - Bagua, 2019. [Tesis de grado]. Chiclayo: Universidad César Vallejo; 2019. [Consultado 10 de enero de 2020] Disponible en: http://repositorio.ucv.edu.pe/bitstream/handle/20.500.12692/43390/Astochado_GTT.pdf? sequence $=1$ \&isAllowed $=\mathrm{y}$ 
Erika María Pabón-Ortíz • Jop Vann-Derth Mora-Cruz • Carmen Yaneth-Buitrago • Ruth Alexandra CastiblancoMontañez

40. Tobar E, Retamal A, Garrido N. Elementos de gestión para un servicio de urgencia de un hospital universitario. Rev. Hosp. Clín. Univ. Chile. [Revista en internet] 2014 [Consultado 10 de enero de 2020]; 25: 189-200. Disponible en: https://www.redclinica.cl/Portals/0/Users/014/14/14/elementos_gestion_servicio_urgencia. pdf

41. Flores-Alpízar CA, Ponce-Moreno R, Ruíz-Jalomo MR, Corral-Quiroz RJ. Factores relacionados con la calidad de atención de enfermería. Rev Enferm Inst Mex Seguro Soc. [Revista en internet] 2014 [Consultado 10 de enero de 2020]; 23(3): 143-148. Disponible en: https://www.medigraphic.com/pdfs/enfermeriaimss/ eim-2015/eim153c.pdf

42. Retamal-Matus H, Aguilar L, Aguilar M, González R, Valenzano S. Factores que influyen en la sobrecarga de los cuidadores de adultos mayores en dos instituciones de la ciudad de Punta Arenas, Chile. Rev. psicogeriatr [Revista en internet] 2015 [Consultado 21 de febrero de 2020]; 5(3): 119-124. Disponible en: https://www. viguera.com/sepg/pdf/revista/0503/503 0119 0124.pdf

43. Carrillo-Algarra AJ, Martínez-Pinto PC, Taborda-Sánchez SC. Aplicación de la filosofía de Patricia Benner para la formación en enfermería. Rev. cuba. enferm. [Revista en internet] 2018 [Consultado 21 de febrero de 2020]; 34(2). Disponible en: http://revenfermeria.sld.cu/index.php/enf/article/view/1522/358

44. De-Arco-Canoles OC. Sobrecarga laboral en profesionales de enfermería de unidades de cuidado intensivo en instituciones hospitalarias de Cartagena de Indias, 2012. [Tesis de grado] Bogotá D.C: Universidad Nacional de Colombia; 2013. [Consultado 15 de marzo de 2020] Disponible en:http://www.bdigital.unal.edu. co/43071/1/5539623.2013.pdf

45. Hernandez-Morquecho KG. Evaluar para gestionar recursos. Análisis de la literatura sobre la carga laboral en enfermería. Rev. enferm. Inst. Mex. Seguro Soc. [Revista en internet] 2016 [Consultado 15 de marzo de 2020]; 24(3): 217-222. Disponible en: https://www.medigraphic.com/pdfs/enfermeriaimss/eim-2016/eim163k.pdf

46. Solis-Muñóz M. Asociación entre la ratio de enfermería y los desenlaces de los pacientes de UCI. Estudio multicéntrico. Enferm Intensiva. 2016; 27(2): 81-83. https://doi.org/0.1016/j.enfi.2016.04.001

47. Kane RL, Shamliyan TA, Mueller C, Duval S, Wilt TJ. The Association of Registered Nurse Staffing Levels and Patient Outcomes: Systematic Review and Meta-Analysis. Med Care. 2007; 45(12): 1195-1204. https:// doi.org/10.1097/MLR.0b013e3181468ca3

48. Fernández-Pinzón YA, Vanegas-Vargas C, Vargas JL. La razón (ratio) enfermera-paciente: Relación con los resultados en los pacientes y esfuerzos por establecerlo por la ley. [Tesis de grado] Bogotá D.C: Universidad Nacional de Colombia; 2009. http:/www.bdigital.unal.edu.co/8495/1/535675.2009.pdf

49. Cabrera-Melo CG, Díaz-Tomas DA, Palacios-Sayritupac VL. Índice Enfermera-Paciente y su relación con la incidencia de neumonías asociadas a ventilación mecánica en las unidades de cuidados intensivos de Lima, 2017. [Tesis de grado] Lima: Universidad Peruana Cayetano Heredia; 2017. [Consultado 05 de abril de 2020] Disponible en: http://repositorio.upch.edu.pe/bitstream/handle/upch/806/Indice CabreraMelo_Carmen.pdf? sequence $=1 \&$ is Allowed $=\mathrm{y}$

50. Monsalve-Prada M. La humanización elemento congruente de la ética aplicada y los eventos adversos en instituciones de salud. Arch Med. 2018; 18 (1): 34-42.https://doi.org/10.30554/archmed.18.1.2524.2018 